\title{
Planning Paths Through Singularities in the \\ Center of Mass Space
}

\author{
William R. Doggett * \\ NASA Langley Research Center, Hampton, Va. 23681-0001 \\ William C. Messner ${ }^{\dagger}$ \\ Carnegie Mellon University, Pittsburgh, Pa. 15251-6224 \\ Jer-Nan Juang ${ }^{*}$ \\ NASA Langley Research Center, Hampton, Va. 23681-0001
}

\begin{abstract}
$\underline{\text { Abstract }}$
The center of mass space is a convenient space for planning motions that minimize reaction forces at the robot's base or optimize the stability of a mechanism. A unique problem associated with path planning in the center of mass space is the potential existence of multiple center of mass images for a single Cartesian obstacle, since a single center of mass location can correspond to multiple robot joint configurations. The existence of multiple images results in a need to either maintain multiple center of mass obstacle maps or to update obstacle locations when the robot passes through a singularity, such as when it moves from an elbow-up to an elbow-down configuration. To illustrate the concepts presented in this paper, a path is planned for an example task requiring motion through multiple center of mass space maps. The object of the path planning algorithm is to locate the bangbang acceleration profile that minimizes the robot's base reactions in the presence of a single Cartesian obstacle. To simplify the presentation, only non-redundant robots are considered and joint non-linearities are neglected.
\end{abstract}

\section{Introduction}

The center of mass $(\mathrm{CM})$ space is the space reachable by a robot's $\mathrm{CM}$ as the robot moves around its workspace. The CM space is important because motion of the robot's CM in the CM space can be directly related to reaction forces at the robot's base. ${ }^{1}$ In addition, location of a mechanism's CM relative to its support points is used to control mechanical stability. ${ }^{2,3}$ Many of the planning techniques applicable to motion in Cartesian space are directly applicable to planning in the CM space. However, the CM space has a unique characteristic related to obstacle locations. A single obstacle in Cartesian space can map to several CM space images, since a single center of mass location can correspond to multiple robot joint configurations. This issue will be explored in depth in this paper.

Copyright (C) 1998 by the American Institute of Aeronautics and Astronautics, Inc. No copyright is asserted in the United States under Title 17, U.S. Code. The U.S. Government has a royalty-free license to exercise all right under the copyright claimed herein for Governmental Purposes. All other rights are reserved by the copyright owner.
Traditionally motion planning has been accomplished in either Cartesian space or robot joint space. ${ }^{4}$ The CM space is a Cartesian like space allowing direct application of many of the existing control techniques. Boulic et. al. demonstrated a resolved rate control scheme applied to the motion of redundant serial kinematic chains containing sixteen or more degrees of freedom. ${ }^{5}$ They also introduced a method for outlining the $\mathrm{CM}$ space. Papadopoulos et al. described a technique for designing a robot with zero base reactions in a limited region of its reachable space, termed the reactionless workspace. ${ }^{6}$ The design was based on an analysis of the motion of the robot's CM. Neither of the above authors investigated path planning. In addition, neither author addressed motion within the $\mathrm{CM}$ space in the presence of obstacles. In Doggett et al. equations describing the path which globally minimizes the robot's base reaction forces while

\footnotetext{
* Mechanical Engineer, System Integration Branch.

* Assistant Professor, Department of Mechanical Engineering.

* Principal Scientist, Structural Dynamics Branch.
} 
Path planning in the presence of obstacles naturally decomposes into planning paths between intermediate goals around the obstacles. Thus a series of segments are planned, which when combined, yield the complete path from the initial configuration to the final configuration. To locate the best path a minimax optimization approach will be used. This is a technique first receiving wide spread use in the signal processing community. The technique seeks to minimize the maximum of a group of functions, in this case the peak reaction forces. Nowrouzian et al. applied minimax optimization to simultaneously meet magnitude and group delay specifications in digital filter design. ${ }^{7}$ Crow et. al. used minimax techniques to locate statistical discontinuities for applications such as traffic monitoring and mechanical failure detection. ${ }^{8}$

This paper will follow a representative planning scenario to illustrate an issue unique to $\mathrm{CM}$ space path planning related to the potential existence of multiple CM space images of a single Cartesian obstacle. The objective of the planning scenario will be to plan paths minimizing the base reaction force while executing a bang-bang acceleration profile. First, the equations will be given for the bang-bang profiles along with a description of the optimization approach used to locate the bang-bang path minimizing the robot's base reaction forces. Second, the issue of mapping the obstacles from Cartesian space to equivalent CM space images will be addressed. Third, a path will be planned for a task that requires the robot to pass through a singularity.

The approach presented assumes the initial positions of the robot joints are given and, during the maneuver, the mass of the robot and the obstacle position remain constant. This paper does not address the issue of smoothing the acceleration profiles between successive paths in an optimal manner. Much work has already been done in this area ( see, for example, the work by Bell. ${ }^{9}$ )). Further, the paper does not address the problem of finding all possible paths from the initial robot configuration to the final CM location. Several techniques have been suggested for developing this information, see Latombe. ${ }^{10}$

\section{Development}

In this section the equations describing the path that the $\mathrm{CM}$ will follow are developed. The equations are formulated assuming that the $\mathrm{CM}$ acceleration profile is bang-bang. The objective is to select the path that minimizes the peak acceleration of the CM during the move. While this is not the global minimum, due to the bang-bang assumption, it could be used as an estimate for further calculations to locate the global minimum.
To formulate the equations, consider motion from $A$ to $C$ over an obstacle with peak at $B$ as illustrated in Fig. 1. The motion comprising a horizontal distance $d$ over an obstacle with height $h_{o b s}$ located at $x_{o b s}$ must be completed in $T$ seconds. The velocity and position at $A$ and $C$ are assumed known. I.e. $v_{x_{A}}, v_{y_{A}}, x_{A}, y_{A}, v_{x_{C}}, v_{y_{C}}, x_{C}$, and $y_{C}$ are known where

$$
\begin{array}{ll}
x_{A}, x_{B}, x_{C} \quad \begin{array}{l}
\text { are the locations along the } x \text { axis at } A, \\
B, \text { or } C \text { respectively, }
\end{array} \\
y_{A}, y_{B}, y_{C} \quad \begin{array}{l}
\text { are the locations along the } y \text { axis at } A, \\
B, \text { or } C \text { respectively, }
\end{array} \\
v_{x_{A}}, v_{x_{B}}, v_{x_{C}} \begin{array}{l}
\text { are the velocities along the } x \text { axis at } \\
A, B, \text { or } C \text { respectively, and }
\end{array} \\
v_{y_{A}}, v_{y_{B}}, v_{y_{C}} \begin{array}{l}
\text { are the velocities along the } y \text { axis at } \\
A, B \text {, or } C \text { respectively, }
\end{array}
\end{array}
$$

Figure 2 depicts a representative pair of bang-bang acceleration profiles. As can be seen from the figure, a single switch from acceleration to deceleration along $x$ can occur. The switch occurs $T_{s x}$ seconds from the initial point at $A$. Two potential switches points exists for the $y$ acceleration occurring at $T_{s y 1}$ or $T_{s y 2}$. The objective is to minimize the Euclidean norm of the acceleration, i.e. $\left\|\left[\begin{array}{ll}a_{x} & a_{y}\end{array}\right]\right\|$, while simultaneously satisfying the boundary conditions at $A$ and $C$. The first step is to locate the switch times by integrating the acceleration profile twice and applying the boundary conditions. The $x$ boundary conditions will be satisfied in the allotted time $T$ by one of two distinct cases.

Case 1) It is possible to move from $A$ to $C$ with a constant acceleration, which may be zero, or

Case 2) an interval of acceleration and deceleration are necessary to move from $A$ to $C$ in the allotted time.

When $a$ is constant, integrating twice leads to the following expression for position $x(t)=\frac{1}{2} a t^{2}+v_{o} t+x_{o}$, where $v_{o}$ and $x_{o}$ are the velocity and position at $t=0$, respectively.

The first case implies

$$
\begin{aligned}
& x_{A C}(t)=\frac{1}{2} a_{x} t^{2}+v_{x_{A}} t+x_{A},\{t \in[0, T]\} \\
& v_{x_{A C}}(t)=a_{x} t+v_{x_{A}},\{t \in[0, T]\}
\end{aligned}
$$

which leads to

$$
\begin{aligned}
& d=\frac{1}{2} a_{x} T^{2}+v_{x_{A}} T+x_{A} \\
& v_{x_{C}}=a_{x} T+v_{x_{A}}
\end{aligned}
$$

Case 1 holds if and only if 


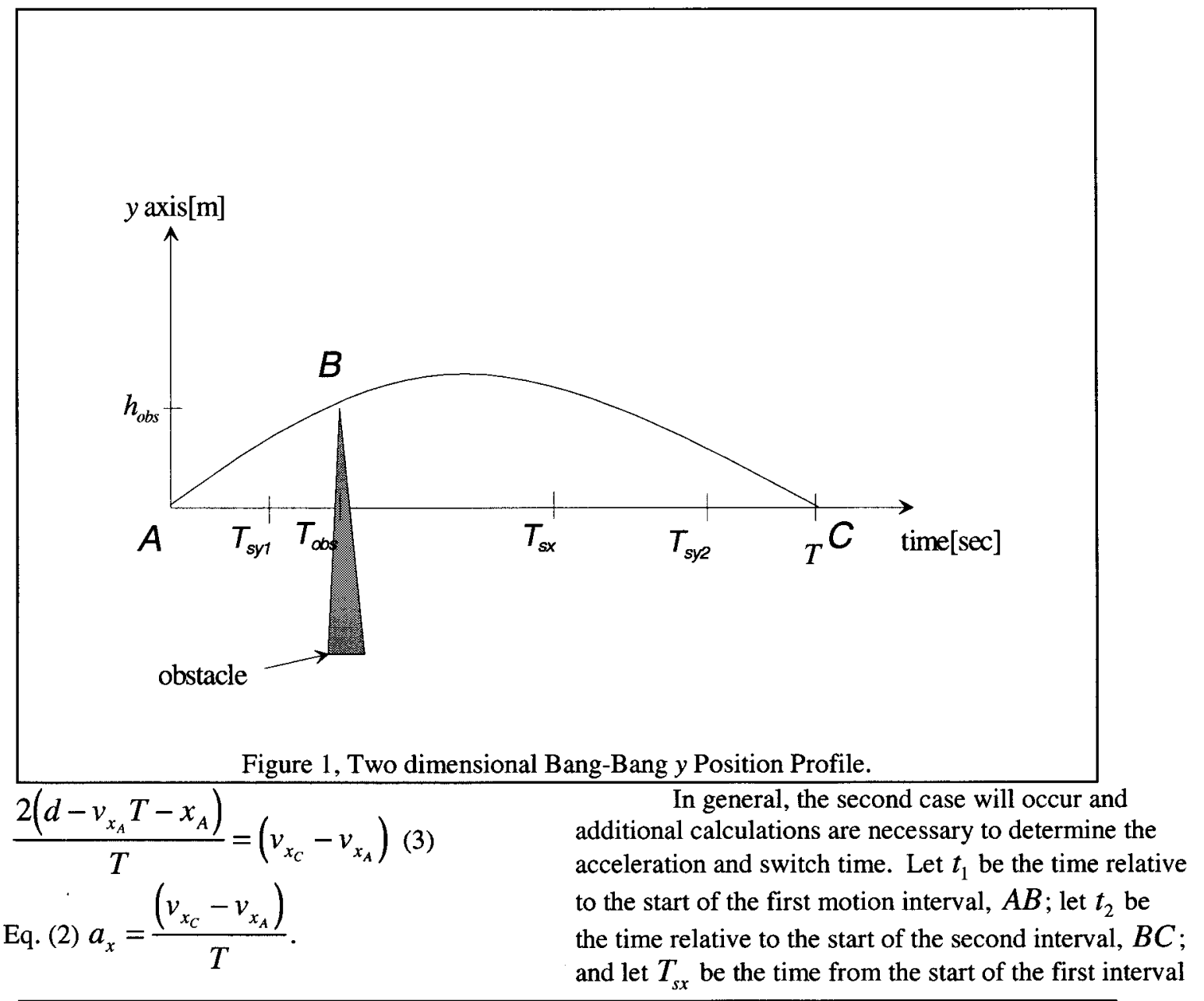

....... y acceleration

$--x$ acceleration

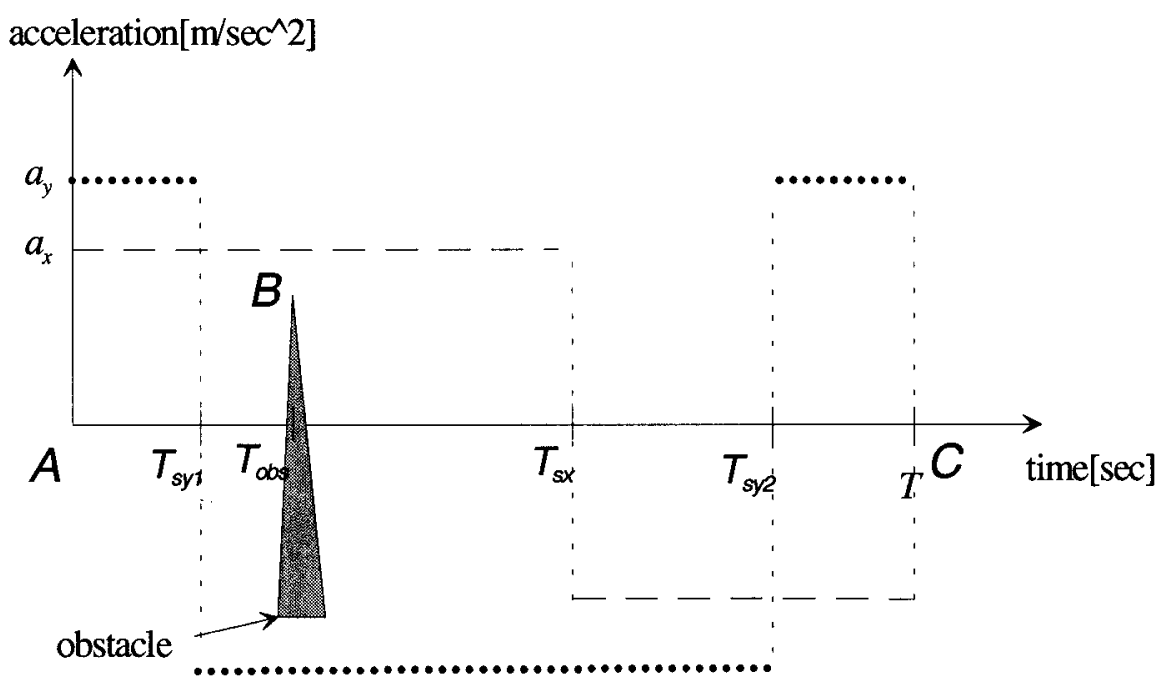

Figure 2, Two dimensional Bang-Bang $x$ and $y$ Acceleration Profile. 
until the switch from acceleration to deceleration occurs. Referring to Fig. 1

$$
\begin{aligned}
& x_{A B}\left(t_{x_{1}}\right)=\frac{1}{2} a_{x} t_{x_{1}}^{2}+v_{x_{A}} t_{x_{1}}+x_{A},\left\{t_{x_{1}} \in\left[0, T_{s x}\right]\right\} \\
& x_{B C}\left(t_{x_{2}}\right)=-\frac{1}{2} a_{x} t_{x_{2}}^{2}+v_{x_{B}} t_{x_{2}}+x_{B} \\
& \quad,\left\{t_{x_{2}} \in\left[0, T-T_{s x}\right]\right\} \\
& x_{B C}\left(T-T_{s x}\right)=d \\
& v_{x_{A B}}\left(t_{x_{1}}\right)=a_{x} t_{x_{1}}+v_{x_{A}},\left\{t_{x_{1}} \in\left[0, T_{s x}\right]\right\} \\
& v_{x_{B C}}\left(t_{x_{2}}\right)=-a_{x} t_{x_{2}}+v_{x_{B}},\left\{t_{x_{2}} \in\left[0, T-T_{s x}\right]\right\} \\
& v_{x_{B C}}\left(T-T_{s x}\right)=v_{x_{C}}
\end{aligned}
$$

Combining Eqs. (4), (5), (6), and (7) leads to

$d=\frac{1}{2} a_{x} T^{2}-a_{x}\left(T-T_{s x}\right)+v_{x_{A}} T+x_{A}$.

Combining Eqs. (7), (8), and (9) leads to

$v_{x_{C}}=-a_{x}\left(T-T_{s x}\right)+a_{x} T_{s x}+v_{x_{A}}$.

Solving Eq. (11) for $a_{x}$ and substituting for $a_{x}$ in Eq.

(10) leads to

$$
\begin{gathered}
\left(T-T_{s x}\right)^{2}\left[-2\left(v_{x_{C}}-v_{x_{A}}\right)\right]+\left(T-T_{s x}\right)\left[4\left(d-v_{x_{A}} T-x_{A}\right)\right] \\
+\left[\left(v_{x_{C}}+v_{x_{A}}\right) T^{2}+2 T\left(x_{A}-d\right)\right]=0
\end{gathered}
$$

which can be solved for $\left(T-T_{s x}\right)$ via the quadratic formula. With $T_{s x}$ known, $a_{x}$ can be calculated using Eq. (10).

Now that the motion in $x$ has been calculated, the equations describing the $y$ motion can be formulated. The time to reach the obstacle, $T_{o b s}$, is set by the motion in the $x$ direction and can be calculated as follows. If the obstacle is encountered during the first acceleration interval, i.e. $T_{o b s} \leq T_{s x}$, then $T_{o b s}$ can be calculated using the known value of $a_{x}$ from $x_{o b s}=\frac{1}{2} a T_{o b s}^{2}+v_{x_{A}} T_{o b s}+x_{A}$. If, however, $T_{o b s}>T_{s x}$, then using Eqs. (4), (5), (6), and (7) leads to

$$
\begin{aligned}
x_{o b s}= & -\frac{1}{2} a_{x}\left(T_{o b s}-T_{s x}\right)^{2} \\
& +\left(a_{x} T_{s x}+v_{x_{A}}\right)\left(T_{o b s}-T_{s x}\right)+\frac{1}{2} a_{x} T_{s x}^{2}+v_{x_{A}} T_{s x}+x_{A}
\end{aligned}
$$

whch can be solved for $T_{o b s}$ via the quadratic formula.

With $T_{o b s}$ known, the derivation described above for the $x$ motion is repeated for each $y$ interval, $A B$ of duration $T_{o b s}$ and $B C$ of duration $T-T_{o b s}$, to find $T_{s y 1}$ and $T_{s y 2}$ of Fig. 2. However, the $y$ velocity at the obstacle top, $v_{y_{s}}$, is unknown. This velocity is determined by minimizing the peak acceleration, $a_{y}$.

The minimization is performed using a minimax optimization strategy with two state variables. The two states correspond to the $y$ velocity at $B$ and the height above the obstacle. The height above the obstacle is a state variable, because it is possible for the initial and final $y$ velocities to be such that moving well above the obstacle is more efficient than passing just above the

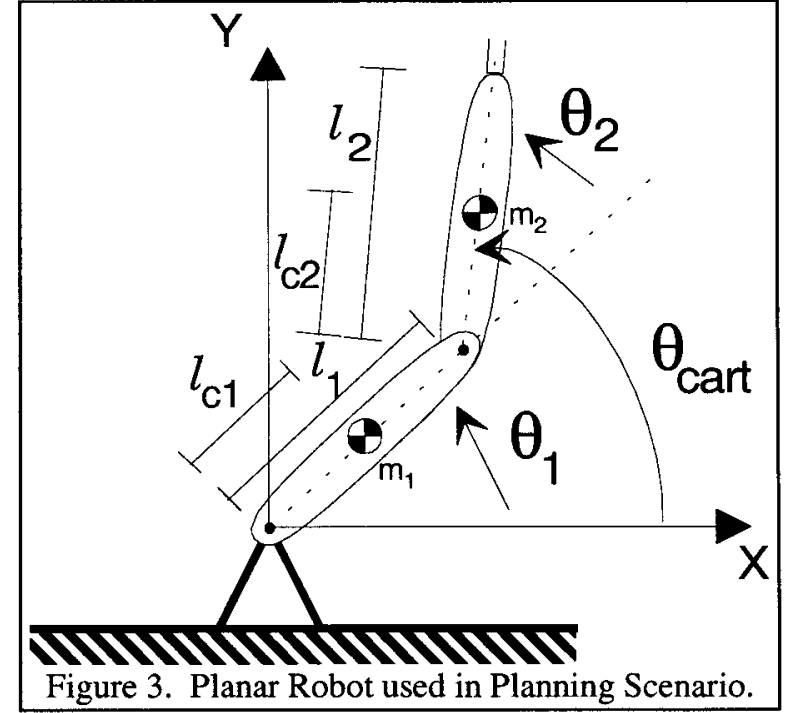

obstacle. A key constraint in the optimization is that each interval must have the same minimum peak acceleration. To illustrate why, consider a case where the first interval's minimum peak acceleration was less than that of the second interval. Then the magnitude of first acceleration profile could be increased, providing more time for the second interval to complete, thus reducing its peak acceleration. This trade off would continue until the optimal motion is determined, where each interval has the same minimum peak acceleration, $a_{\mathrm{y}}$.

\section{Representative Planning Scenario}

In this section a representative planning scenario will be used to illustrate a unique issue related to $\mathrm{CM}$ space path planning. The scenario is based on the two degree of freedom (DOF) robot with revolute joints shown in Fig. 3 . The quantities $l_{1}, l_{2}, l_{c 1}, l_{c 2}, m_{1}$ and $m_{2}$ are the link length, location of the link CM along the link, and link mass for joints 1 and 2 ,

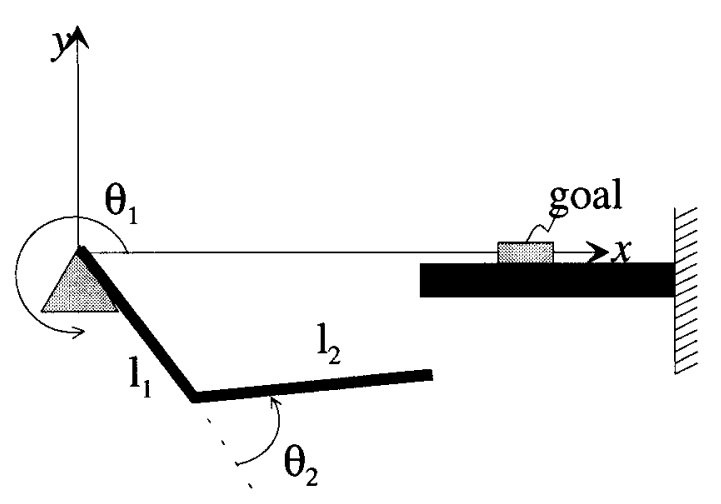

Figure 4. Initial Robot Configuration and Goal Requiring Motion through Singularity (i.e. to CM Space Boundary). 


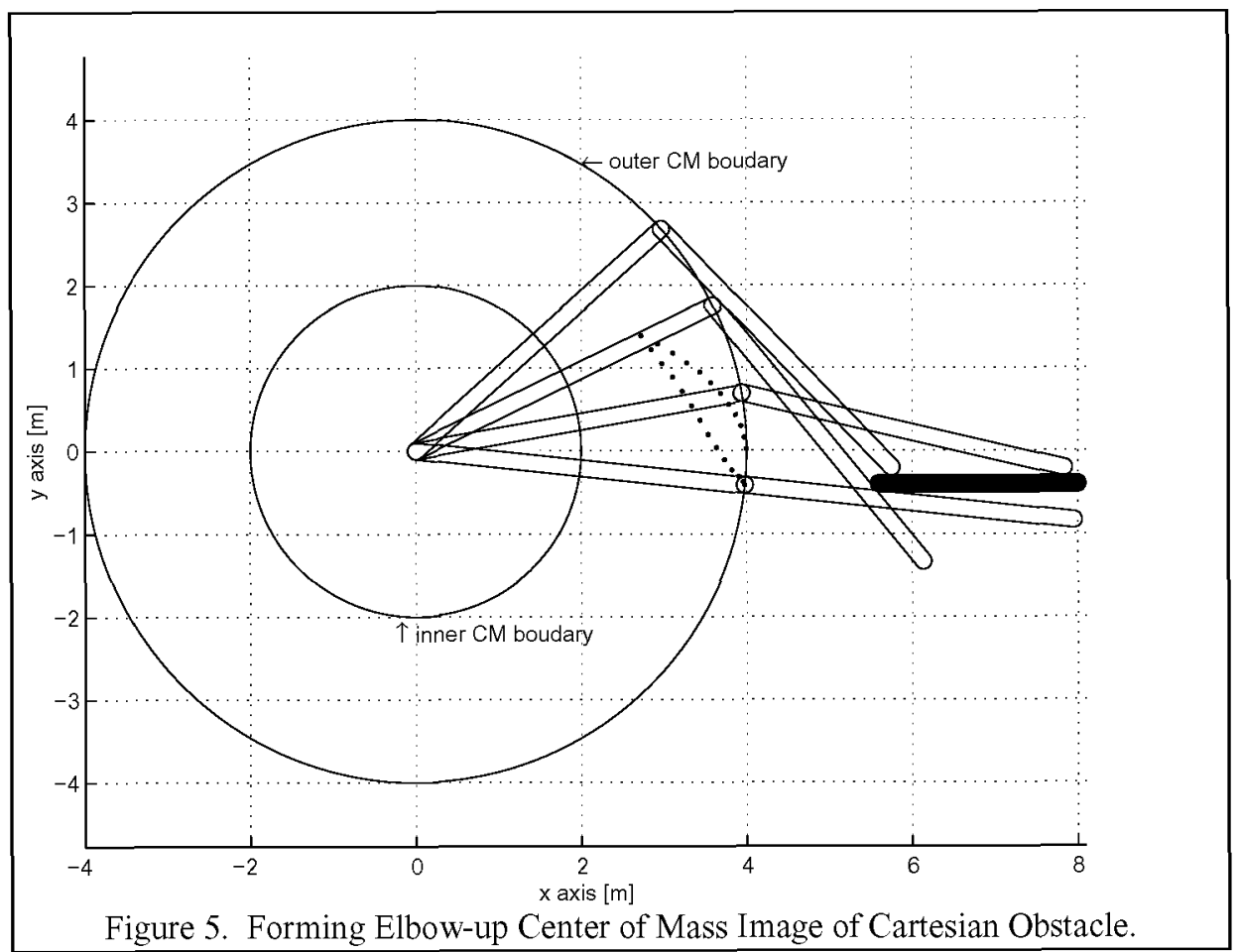

the right. The objective is to plan a path from the robot configuration shown to a configuration where the robot's end point is just touching the box at the top center identified by "goal". The first step in CM space path planning is to map the obstacles into the CM space by sliding the robot around the obstacle and noting the location of the robot's CM as shown in Fig. 5. Figure 5 was constructed based on the following values:

$$
\begin{aligned}
& l_{1}=l_{2}=4[\mathrm{~m}] \\
& m_{1}=m_{2}=\frac{1}{2}[\mathrm{~kg}] \\
& l_{c 1}=l_{c 2}=\frac{l_{1}}{2}=2[\mathrm{~m}] \\
& T=1[\mathrm{sec}]
\end{aligned}
$$

In Fig. 5 the two circles correspond to the inner and outer CM boundaries and the dots represent the robot $\mathrm{CM}$ location for a given arm configuration. The reachable CM space is the area between the two concentric circles. The inner circle is formed by folding the robot over on itself, such that $\theta_{2}=180$, and moving the first joint through one complete revolution, $\theta_{1}: 0^{\circ} \rightarrow 180^{\circ}$. The outer circle is formed in a similar fashion with the robot fully extended, $\theta_{2}=0^{\circ}$. For the purpose of this example joint range limits have been ignored. However, if range limits existed, this would have no affect on the algorithm presented. Range limits only affect the shape of CM space.

Notice that the robot is maintaining the elbowup configuration during the formation of the CM image of Fig. 5. (Elbow-up is defined as the robot configuration corresponding to location of joint 2 on the left side of a vector originating at joint 1 and passing through the robot end-point as viewed looking along the vector from a point above, positive $Z$ in the figure, the origin.) Alternatively, the robot could maintain an elbow-down configuration. This leads to two potential CM space images for the table of Fig. 4 corresponding to the two robot parities as shown in Fig. 6 . If additional obstacles were present in the robot's Cartesian workspace, each obstacle could be mapped individually, then composite CM space maps would be formed from a union of the elbow-up and elbow-down images, respectively. This approach would enable a planner to add more detail as necessary. In this case, only collisions with the table are considered, however it is also important not to pass through the box while moving to the goal. It will be shown that collision checks with box are unnecessary because the optimum path for this problem approaches the box from above, completely avoiding contact with the box.

The planning problem can be viewed in the CM space as moving from the darkened circle labeled $A$ at the bottom of Fig. 7 to the square labeled $E$. In Fig. 7 the small circles represent the initial configuration of the robot, while the small squares identify the potential final configurations. The darkened circle and square identify the elbow-down configurations for the initial and final configuration, respectively. Notice that the final elbow-down configuration is completely enclosed in the elbow-down obstacle image. This indicates that a collision with the obstacle would occur before the robot was able to reach the box in the elbow-down configuration. Thus, the robot must approach the top of the box in an elbow-up configuration. 


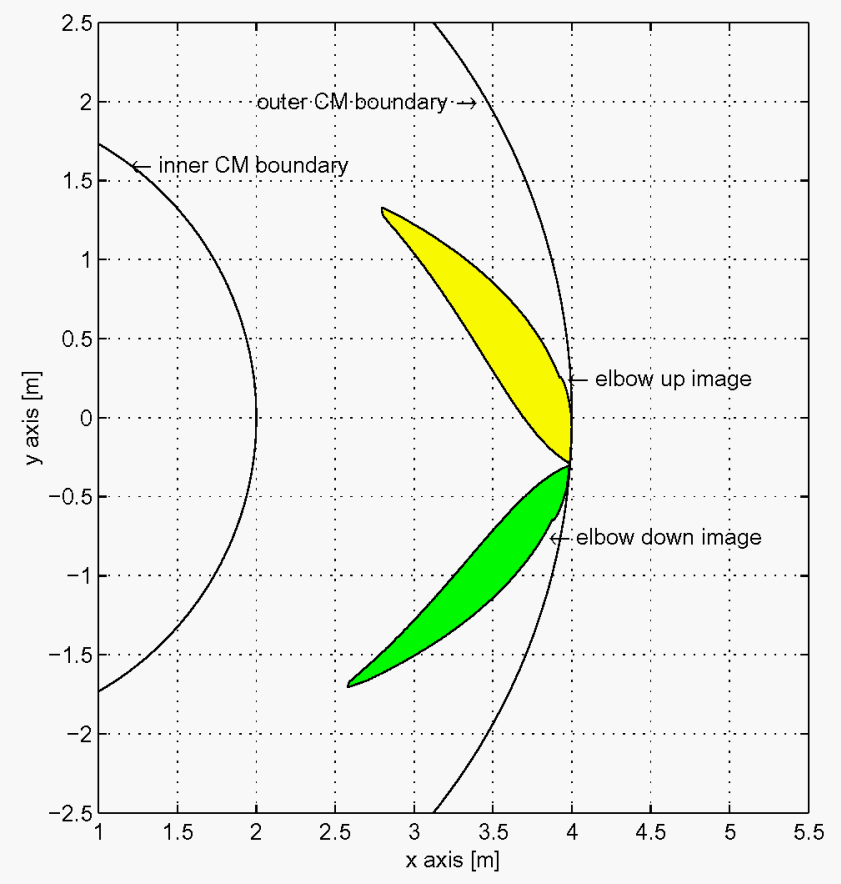

Figure 6. Multiple CM Space Images of Single Cartesian Obstacle.

The next question is how can the information about the two CM images of the obstacle be used. One idea is to recombine the two images into a single image either by a union of each image in the CM space or by enclosing both images within a single object. It will be shown in a representative scenario that either approach is unacceptable, because each artificially restricts the reachable $\mathrm{CM}$ space. Instead, in the case of the $2 \mathrm{DOF}$ robot, two CM space maps must be maintained. The two maps can be viewed as two surfaces of a planar

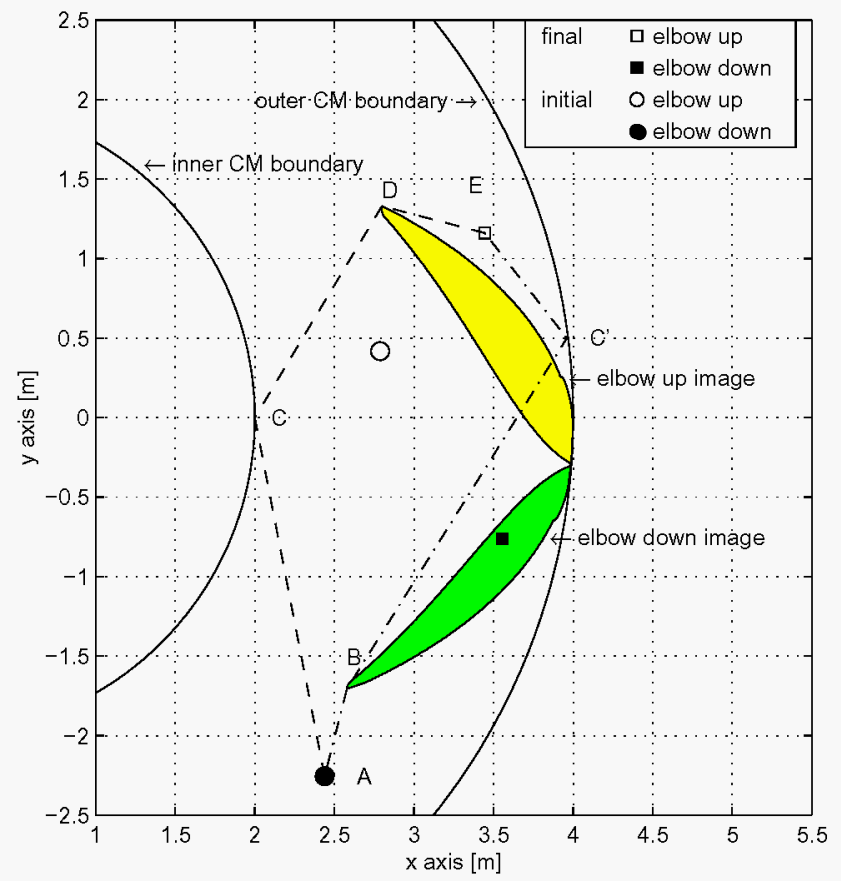

Figure 7. Two Possible CM Space Paths to Final Configuration 
annulus. ${ }^{\S}$ One, the top, represents the elbow-up robot map or elbow-up robot parity, the other the elbowdown parity. Transitions between the two maps can only occur at the radial boundaries of the annulus. Figure 6 is a view from above the transparent annulus allowing simultaneous viewing of both obstacles.

There are two potential strategies for moving from the initial elbow-down configuration to the final elbow-up configuration. Each requires motion through a singularity either at the inner or outer CM space boundary. The first strategy is to fold the robot completely over on its self $\left(\theta_{2}=180^{\circ}\right)$, moving the robot's $\mathrm{CM}$ to the inner $\mathrm{CM}$ space boundary. The second strategy is to fully extend the robot $\left(\theta_{2}=0^{\circ}\right)$, moving the robot's $\mathrm{CM}$ to the outer $\mathrm{CM}$ space boundary. This second strategy would be completely overlooked if only a single CM map was maintained.

The following scheme is used to locate the optimum bang-bang path to the inner boundary. Referring to the dashed line in Fig. 7, the objective is to go from $A$ to some point on the inner CM boundary, denoted by $C$, and then over the elbow-up image peak, indicated by $D$, to $E$. The optimization is formulated as a minimax problem, where the objective is to minimize the maximum acceleration along each sub-path, $A C$ and $C E$. Each sub-path is located using the method described in Sec. 2. The boundary conditions for the overall move are that the initial and final velocities are zero, while the initial position corresponds to $A$ and the final position corresponds to $E$. At position $C$, continuity in position and velocity are imposed.

To reduce the dimension of the state space searched by the optimization procedure, several characteristics of the problem geometry can be exploited. First, the inner boundary is a circle of radius 2 , and thus the $y$ coordinate of $C$ uniquely identifies $C$ s location in the reasonable search region. Second, the singularity condition at the inner boundary forces the velocity at $C$ to be tangent to the inner circle. Therefore the optimization attempts to minimize the maximum acceleration by determining the optimal values of the following state variables: (i) the time from $A$ to $C$, (ii) the $y$ coordinate of $C$, and (iii) the magnitude of the tangent velocity at $C$.

The resulting trajectory is shown in Fig. 8 by the dashed line. The elbow-up section is indicated by

\footnotetext{
\$ A washer, as in a bolt, nut, and washer.

** A singular configuration is a robot

configuration that reduces the useable degrees of freedom of the robot. For example, with the robot fully extended, $\theta_{2}=0^{\circ}$, end point motion is only possible perpendicular to the axis of the final link, $l_{2}$. Thus the 2 DOF robot of Fig. 3, has only 1 DOF, in this configuration, i.e. motion along the tangent line.
}

open triangles while darkened inverted triangles are used to indicate the elbow-down section of the path. This path results in a peak acceleration of $26.8 \mathrm{~m} / \mathrm{s}^{2}$. The final values for the three state variables indicated that the time to move from $A$ to $C$ was 0.45 seconds while the time to move from $C$ to $E$ was 0.55 seconds. The tangent velocity at $C$ was $-6.3 \mathrm{~m} / \mathrm{s}^{2}$ and the path intersects the inner circle at a $y$ coordinate of $0.1 \mathrm{~m}$.

While joint limits have been ignored in this example, the joint positions, velocities, and accelerations must be realistic, especially as the robot moves through the singularity. Figures 9 and 10, are plots of the joint positions and velocities, respectively. The joint positions appear smooth and the joint velocities are finite at the singularity. The step change in Fig. 9 at approximately 5.5 seconds is due to a wrapping from $2 \pi$ to $-2 \pi$. The sharp transitions in the joint velocities of Fig. 10 are expected from the bangbang acceleration profiles. Optimal smoothing of these transitions was not addressed in this paper, however other researchers have suggested potential schemes. ${ }^{9}$

The second possible strategy is to move from $A$ of Fig. 7 over the elbow-down image at $B$ to the outer $\mathrm{CM}$ boundary, indicated by $C$, and then to $E$ avoiding the elbow-up image. As in the first strategy, we are able to take advantage of the geometry to reduce the state space to the same three variables used above. The trajectory resulting from the minimax optimization is shown in Fig. 11. Notice that the path appears to move through the elbow-up image, however this portion of the path is executed on the bottom of the annulus where only the elbow-down image is present. The elbow-up image becomes important when the CM reaches the outer CM space boundary, and the planner begins to use the elbow-up CM map, avoiding the elbow-up image, as the robot moves to $E$. The final values for the three state variables indicated that the time to move from $A$ to $C^{n}$ was 0.7 seconds while the time to move from $C^{n}$ to $E$ was 0.3 seconds. The tangent velocity at $C$ was 3.0 $\mathrm{m} / \mathrm{s}^{2}$, and the path intersects the inner circle at a $y$ coordinate of $0.68 \mathrm{~m}$. This path results in a uniform peak force of $23.2 \mathrm{~N}$ at the base of the $1 \mathrm{~kg}$ robot, which is considerably better than the $26.8 \mathrm{~N}$ of the path to the inner boundary. It is important to remember that if two separate obstacle maps had not been maintained, then this path would not have been located. While this is not catastrophic in this situation, it could be if the path to the inner boundary was blocked by another obstacle. If the inner boundary path is blocked and only a single CM space map is maintained, then the task would be erroneously labeled infeasible. 


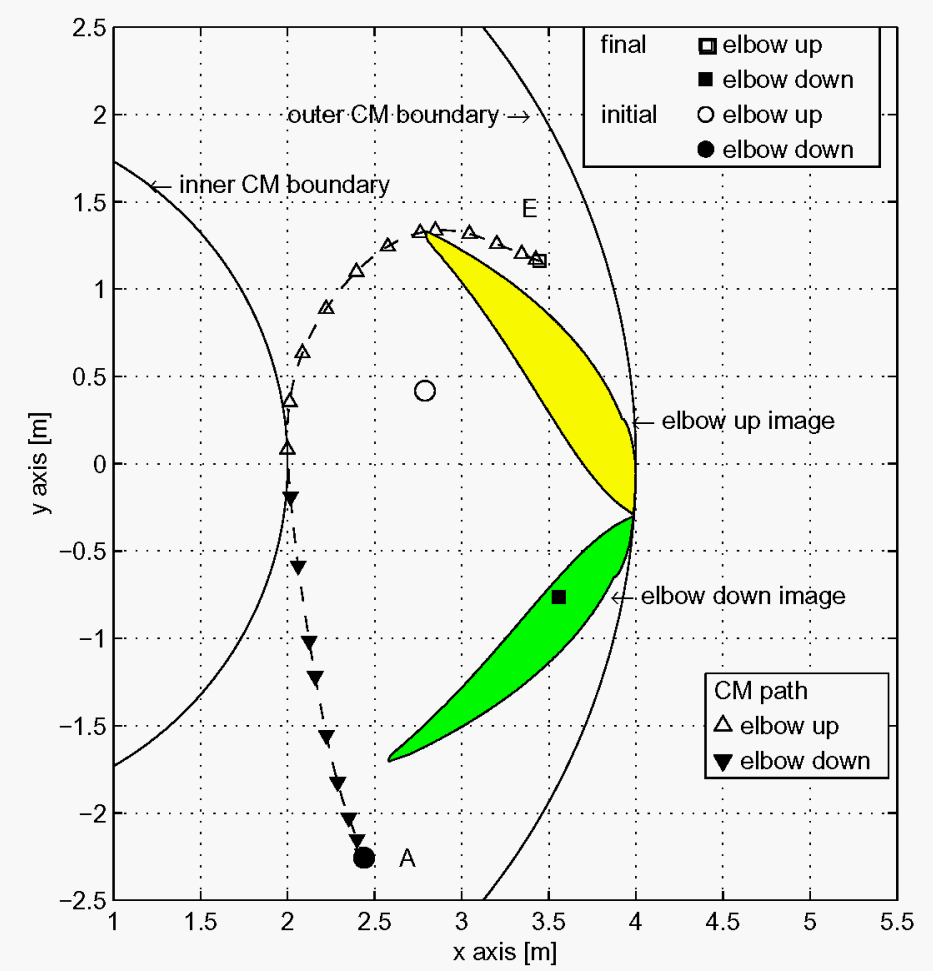

Figure 8. Optimum Bang-Bang Path to Inner Boundary

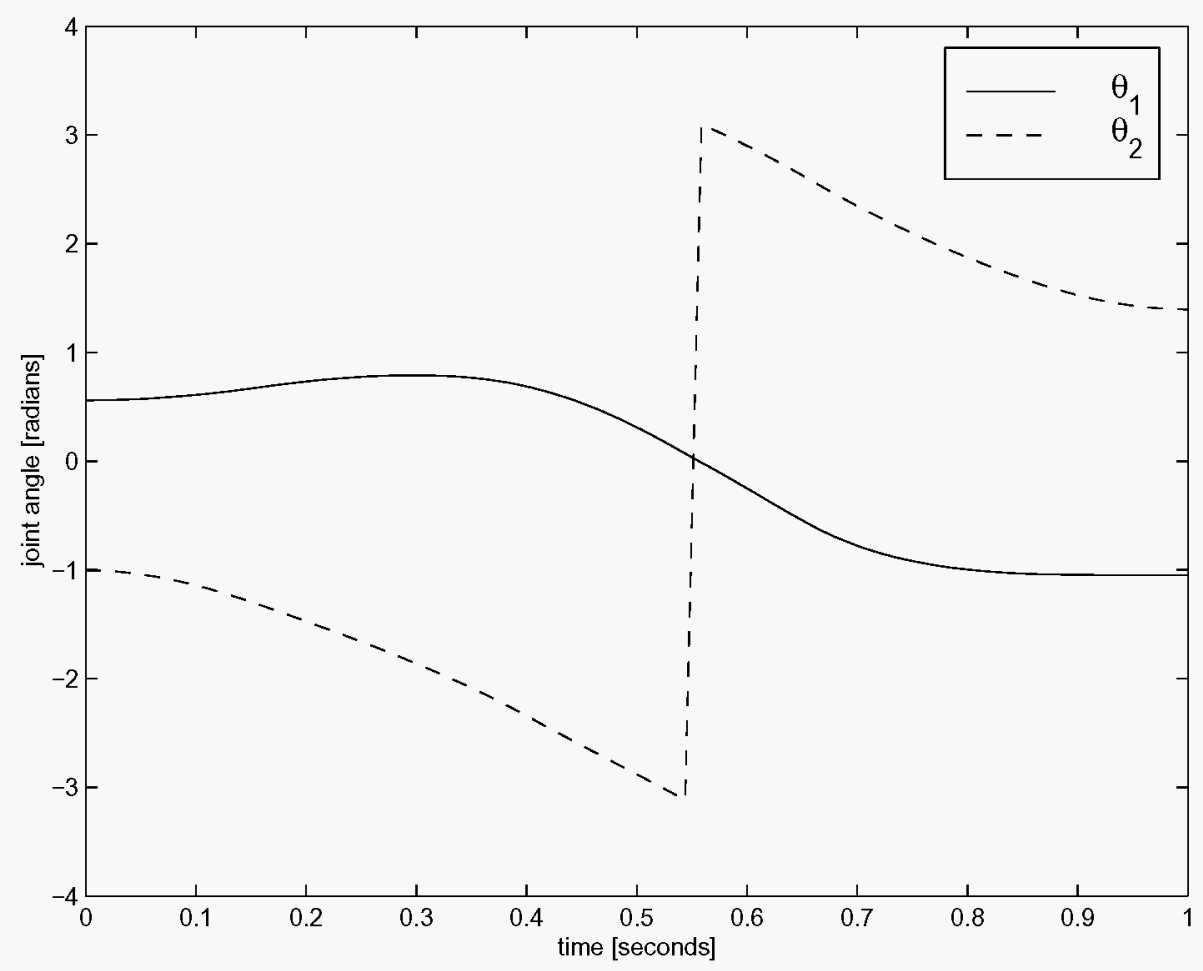

Figure 9. Joint Positions for Motion to Inner Boundary

American Institute of Aeronautics and Astronautics 


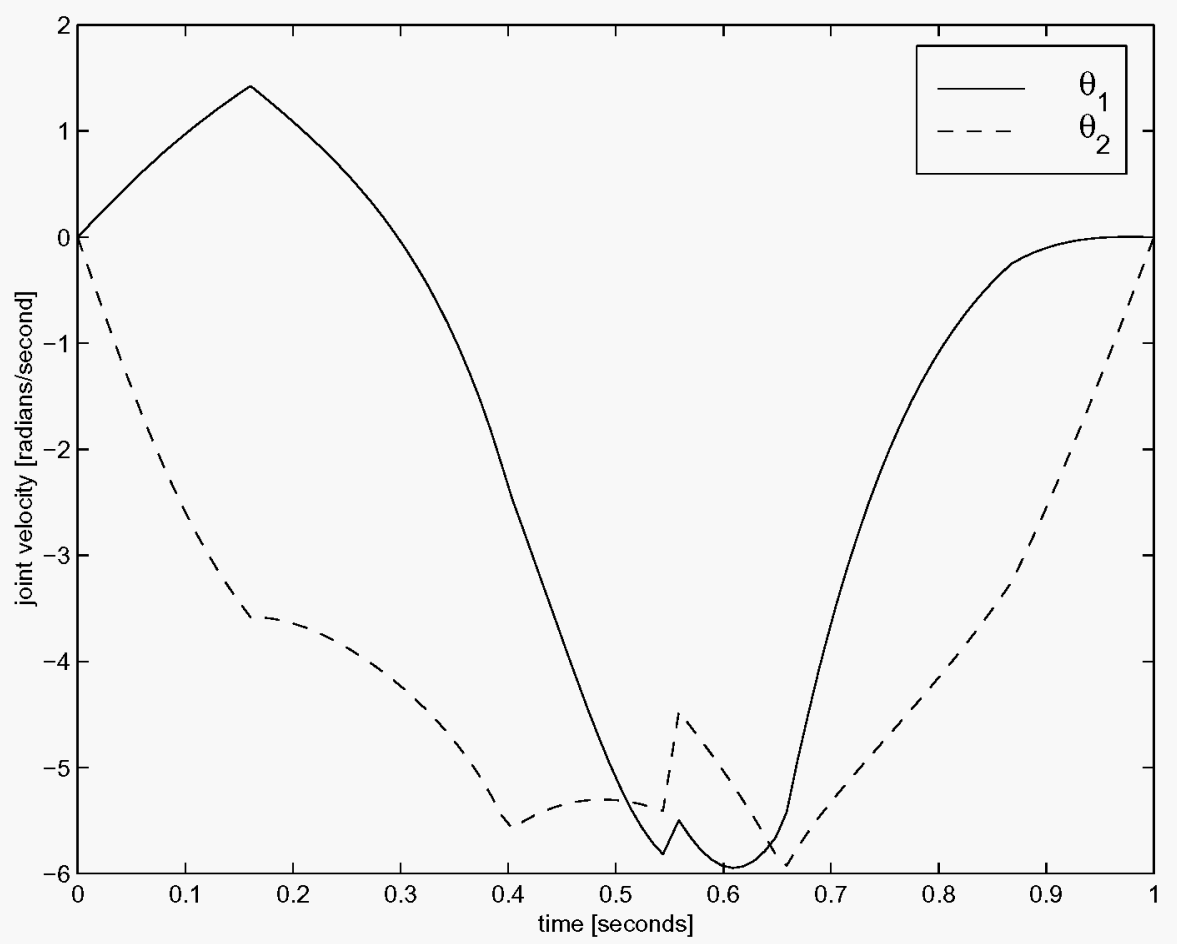

Figure 10. Joint Velocities for Motion to Inner Boundary

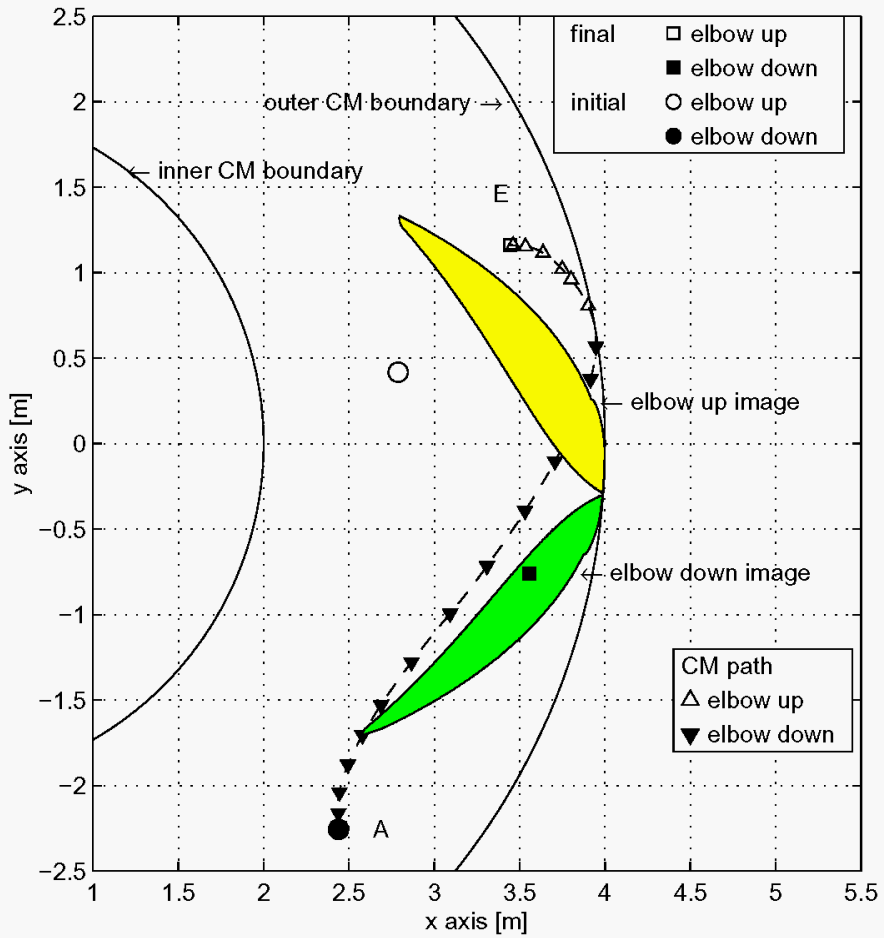

Figure 11. Optimum Bang-Bang Path to Outer Boundary

American Institute of Aeronautics and Astronautics 


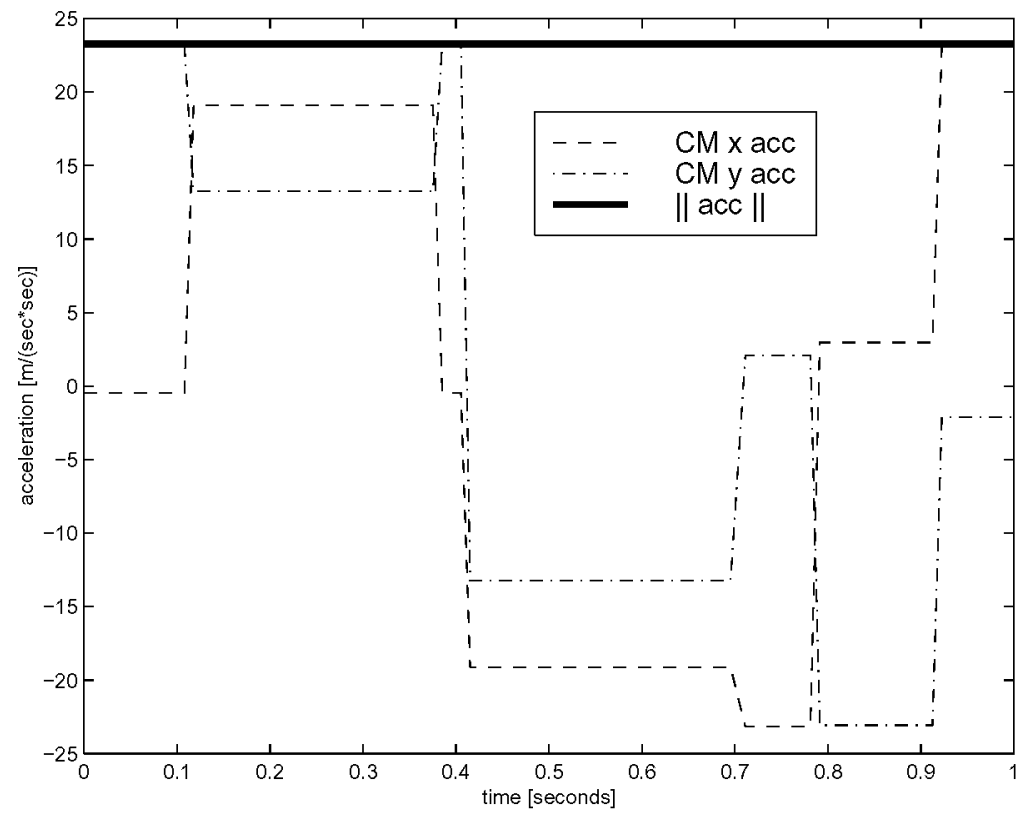

Figure 12. CM Acceleration for Optimum Bang-Bang Path to Outer Boundary accelerations are used to calculate the joint accelerations. As shown in Fig. 13, the peak torque occurs in joint 1 and is approximately equal for both motions. The high torque at the end of the motion to the outer boundary is due to the necessity of maneuvering with the robot nearly fully extended when its rotational inertia is maximal. Figure 14 shows the superiority of the motion to the outer boundary in terms of peak joint 2 torque. The motion to the inner boundary requires joint 2 to move through more than 270 degrees compared to less than 90 degrees for the
The CM acceleration profile for the path of Fig. 11 is shown in Fig. 12. Notice that the Euclidean norm of the $(x, y)$ acceleration is a constant $23.2 \mathrm{~m} / \mathrm{s}^{2}$ as expected from the bang-bang assumption. Note that the $x$ and $y$ acceleration profiles do not appear to be moving (banging) between two constant extreme acceleration values. This is because the bang-bang acceleration profiles are calculated relative to the line segments $A C^{n}$ and $C^{2} E$ of Fig. 11. The accelerations of Fig. 12 are relative to the $x$ and $y$ axes of Fig. 11 .

While no attempt was made to optimize the joint torques, a comparison shows that the path to the outer boundary is slightly better than the path to the inner boundary. The joint torques required to execute the paths can be calculated after the known CM

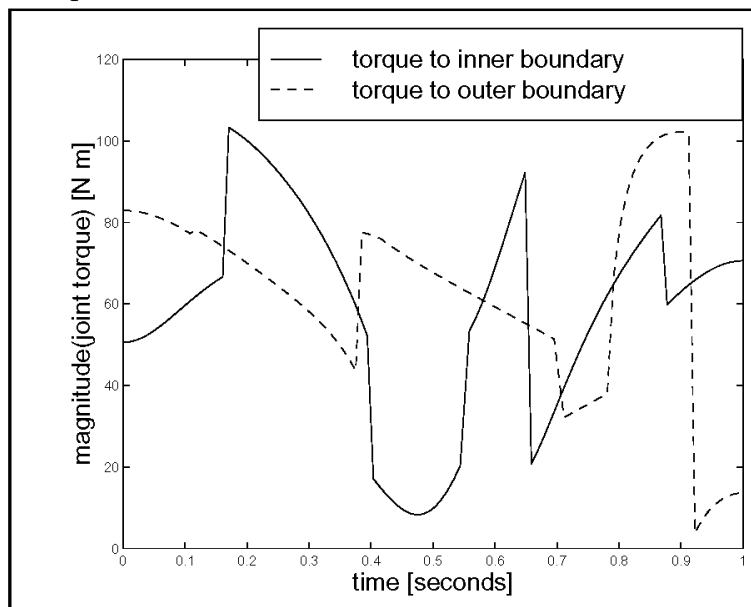

Figure 13. Joint 1 Torque for paths to Inner and Outer CM space boundaries motion to the outer boundary, resulting in the higher joint torque profile shown in Fig. 14.

Figures 15 and 16 display the robot motions corresponding to the paths to the inner and outer CM space boundaries shown in Figs. 8 and 11, respectively. In Figs. 15 and 16, the obstacle is shown in black while a solid line indicates the motion of the robot tip. The motion to the outer boundary appears to be more efficient as expected from the comparison of the peak acceleration along each path.

\section{Conclusions}

In this paper the effect of singularities on the planning process in the center of mass (CM) space has

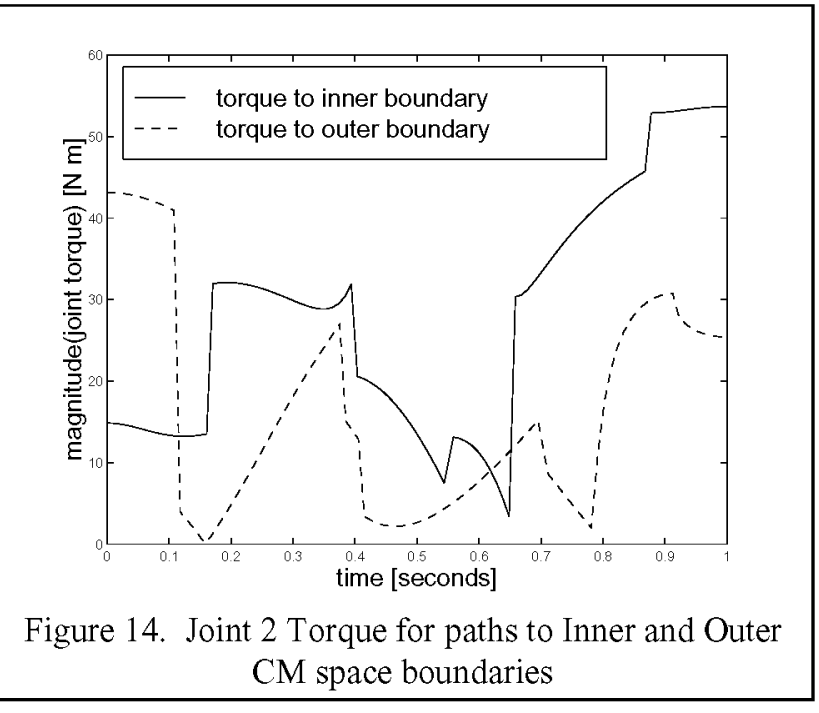




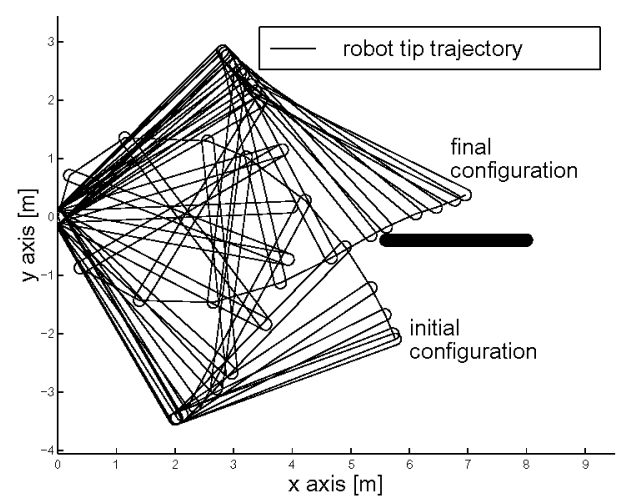

Figure 15. Robot Motion to Inner Boundary

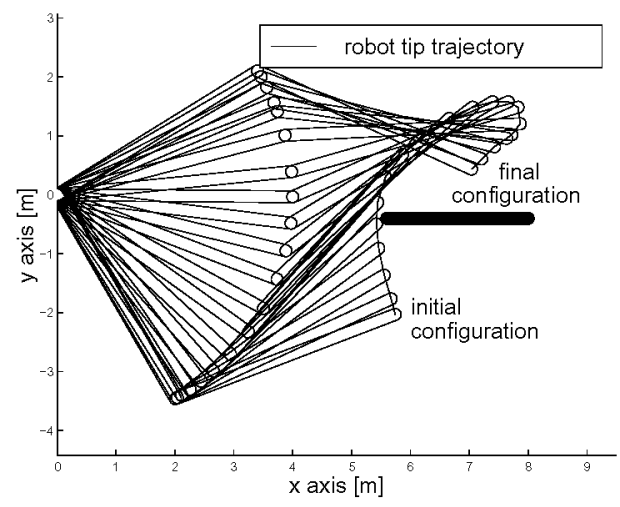

Figure 16. Robot Motion to Outer Boundary

been investigated. The CM space is a Cartesian-like space useful for planning paths that minimize robot base reaction forces or maintain the stability of a mechanism. This paper has introduced a problem unique to $\mathrm{CM}$ space path planning. The problem stems from the existence of multiple CM space images of a single Cartesian obstacle. As shown in a representative planning scenario, existence of multiple images necessitates maintenance of multiple CM space maps, each map specific to a particular robot parity, for example elbow-up vs. elbow-down. To begin the planning process a unique map is selected based on initial robot parity. If singularities exist in the workspace, then path to/through the singularities must be planned and alternate $\mathrm{CM}$ maps explored. The minimal path is then determined by selecting the optimal path from the set of all complete paths found.

This process was examined in detail through a planning scenario based on a two-degree-of-freedom robot avoiding an obstacle. The objective was to locate the bang-bang path that resulted in the minimum base reaction force while accomplishing a task. It was shown that it was necessary to maintain multiple CM space maps for effective planning, each specific to a particular robot parity. Failure to maintain separate CM space maps artificially reduced the number of potential strategies that could be applied to solve the specified task. At best this would reduce the efficiency with which the task was accomplished, as illustrated by the representative planning scenario. At worst the task could be erroneously labeled unfeasible.

References

1 Doggett, W.; Juang, J.N.; and W. Messner. "Global Minimization of Robot Base Reaction Forces During Point to Point Moves", AIAA Guidance Navigation and Control Conference, July 1996, AIAA paper \# 96-3896.

2 Kang, Dong-Oh; Lee, Yun-jung; Lee, Seung-ha; Hong, Yeh Sun; and Zeungnam Bein. "A Study on an Adaptive Gait for a Quadruped Walking Robot under External Forces", Proceedings of the 1997 IEEE International Conference on Robotics and Automation, pp 2777- 82.

3 Kajita, Shuuji and Kazuo Tani. "Study of Dynamic Biped Locomotion on Rugged Terrain", Proceedings of the 1991 IEEE International Conference on Robotics and Automation, pp 140511.

4 John J. Craig. Introduction to Robotics Mechanics and Control, Addison-Wesley Publishing Company, Inc. 1986.

5 Boulic, Ronan; Mas, Ramon; and Daniel Thalmann. "Interactive Identification of the Center of Mass Reachable Space for an Articulated Manipulator", International Conference on Advanced Robotics, 1997, pp. 589-94.

6 Papadopoulos, Evangelos and Ahmed Abu-Abed. "Design and Motion Planning for a Zero-Reaction Manipulator", IEEE International Conference on Robotics and Automation, 1994, Vol. 2, pp 1554-9.

7 Nowrouzian, Behrouz and Balakrishnan Iyer. "Constrained Min-Max Optimization of Multi-Rate Digital Filters with Simultaneous Magnitude and Group-Delay Specifications", Proceedings of $37^{\text {th }}$ Midwest Symposium on Circuits and Systems, 1994, pp. 975-8, Vol. 2.

8 Crow, Robert and Stuart Schwartz. "On Robust Quickest Detection Procedures", Proceedings of the 94 IEEE international Symposium on Information Theory, pg. 258.

9 Bell, Mark J. and John L. Junkins. "Near Minimum-Time Three Dimensional Maneuvers of Rigid and Flexible Spacecraft", The Journal of the Astronautical Sciences, Vol. 42, No. 4, Oct.-Dec. 1994, pp. 421-438.

10 Jean-Claude Latombe. Robot Motion Planning, Kluwer Academic Publishers, Boston, Ma. 1996. 